\title{
Study of Higgs effective couplings at electron-proton colliders
}

\author{
Hoda Hesari, ${ }^{1, *}$ Hamzeh Khanpour, ${ }^{2,1, \uparrow}$ and Mojtaba Mohammadi Najafabadi ${ }^{1, \$}$ \\ ${ }^{1}$ School of Particles and Accelerators, Institute for Research in Fundamental Sciences (IPM), \\ P.O. Box 19395-5531, Tehran, Iran \\ ${ }^{2}$ Department of Physics, University of Science and Technology of Mazandaran, \\ P.O. Box 48518-78195, Behshahr, Iran
}

(Received 24 June 2017; revised manuscript received 7 March 2018; published 30 May 2018)

\begin{abstract}
We perform a search for beyond-the-Standard-Model (BSM) dimension-six operators relevant to the Higgs boson at the Large Hadron Electron Collider (LHeC) and the Future Circular Hadron Electron Collider (FCC-he). With a large amount of data $\left(\mathrm{few} \mathrm{ab}^{-1}\right.$ ) and collisions at the $\mathrm{TeV}$ scale, both $\mathrm{LHeC}$ and FCC-he provide excellent opportunities to search for the BSM effects. The study is done through the process $e^{-} p \rightarrow h j \nu_{e}$, where the Higgs boson decays into a pair of $b \bar{b}$, and we consider the main sources of background processes, including a realistic simulation of detector effects. For the FCC-he case, in some signal scenarios, to obtain an efficient event reconstruction and to have a good background rejection, jet substructure techniques are employed to reconstruct the boosted Higgs boson in the final state. In order to assess the sensitivity to the dimension-six operators, a shape analysis on the differential cross sections is performed. Stringent bounds are found on the Wilson coefficients of dimension-six operators with the integrated luminosities of $1 \mathrm{ab}^{-1}$ and $10 \mathrm{ab}^{-1}$, which in some cases show improvements with respect to the high-luminosity LHC results.
\end{abstract}

DOI: 10.1103/PhysRevD.97.095041

\section{INTRODUCTION}

So far, the Standard Model (SM) of particle physics has been found to be a successful theory describing nature up to the electroweak scale. However, there are reasons to believe that the SM is not the ultimate theory of particle physics at the TeV scale. The Higgs boson discovery by the LHC experiments $[1,2]$ has been a milestone in understanding the mechanism of electroweak symmetry breaking (EWSB). After that, one of the main goals would be to precisely measure the Higgs boson properties that would provide the possibility of searching for new physics effects beyond the SM.

Given the absence of any signature of new physics in the present data, one can parametrize the effects of beyond-the$\mathrm{SM}$ in an effective field theory (EFT) expansion. This approach is a powerful tool that parametrizes possible new physics effects via a systematic expansion in a series of higher-dimensional operators composed of SM fields [3,4].

\footnotetext{
*h.hesari@ipm.ir

${ }^{\dagger}$ Hamzeh.Khanpour@mail.ipm.ir

*mojtaba@ipm.ir
}

Published by the American Physical Society under the terms of the Creative Commons Attribution 4.0 International license. Further distribution of this work must maintain attribution to the author(s) and the published article's title, journal citation, and DOI. Funded by SCOAP.
The operators are composed of all possible combinations of SM fields respecting the $S U(3)_{c} \times S U(2)_{\mathrm{L}} \times U(1)_{\mathrm{Y}}$ gauge symmetries and Lorentz invariance. In the EFT approach, potential deviations from the SM could be described using the following Lagrangian:

$$
\mathcal{L}_{\mathrm{SMEFT}}=\mathcal{L}_{\mathrm{SM}}+\sum_{i} \frac{c_{i}}{\Lambda^{2}} \mathcal{O}_{i}+\text { H.c. },
$$

where $\mathcal{O}_{i}$ is the $i$ th dimension-six operator, $\Lambda$ is the scale at which new physics is expected to appear, and $c_{i}^{\prime}$ 's are arbitrary Wilson coefficients. These dimension-six operators have been listed and studied in Refs. [3-6]. There have been many studies that probe these operators, and so far much work has been devoted to constraining these operators, which can be found in Refs. [7-33].

The aim of this study is to explore Wilson coefficients of dimension-six operators, as described in Refs. [34-36], contributing to the Higgs production in association with a jet and a neutrino at the LHeC and FCC-he [37-40]. The $\mathrm{LHeC}$ is a proposed deep inelastic electron-nucleon scattering (DIS) machine, which has been designed to collide electrons with an energy from $60 \mathrm{GeV}$ to possibly $140 \mathrm{GeV}$ with protons with an energy of $7 \mathrm{TeV}$. The future circular collider (FCC) has the option of colliding electrons with protons with an electron energy $E_{e}=60 \mathrm{GeV}$ and a proton energy of $E_{p}=50 \mathrm{TeV}$. The inclusive Higgs boson production cross section at the high-energy FCC-he is 
expected to be about 5 times larger than at the future proposed high-energy and high-luminosity electronpositron collider TLEP/FCC-ee [41]. In comparison with the LHC or FCC-hh, the LHeC or FCC-he have the advantage of providing a clean environment with small background contributions from QCD strong interactions. Furthermore, no effects from pileup or multiple interactions exist in these machines, and they are able to provide precise measurements of the proton structure, and electroweak and strong interactions.

This paper is structured as follows: In Sec. II, we present the theoretical framework of our analysis by recalling the relevant aspects of the effective SM in which dimension-six operators are considered. We review the higher-dimensional operators and highlight the operators contributing to the Higgs boson production processes at the LHeC and FCC-he. Section III describes the details of our analysis, including the simulation tools and analysis strategy for both $\mathrm{LHeC}$ and FCC-he. We explain the event selection criteria and statistical method by which we obtain the constraints on the Wilson coefficients. The analysis strategy for the $\mathrm{LHeC}$ collider and its sensitivity to the dimension-six operators is presented in Sec. IV. Section V is dedicated to presenting the sensitivity of the FCC-he collider to the related Wilson coefficients in the $h j \nu_{e}$ process. Our results and the constraints on the Wilson coefficients are given in Sec. VI. Comparisons with the LHC bounds are also made in this section. Finally, Sec. VII presents the summary and conclusions.

\section{THEORETICAL FRAMEWORK}

The SM effective Lagrangian can be obtained by including higher-dimensional operators that take into account the new physics effects beyond the SM which may appear at the energy scale much larger than the SM energy scale. Under the assumption of baryon and lepton number conservation and keeping only dimension-six operators, the most general $S U(3)_{\mathrm{C}} \times S U(2)_{\mathrm{L}} \times U(1)_{\mathrm{Y}}$ gauge-invariant Lagrangian can be constructed from the SM fields. We concentrate on the dimension-six interactions of the Higgs boson, fermions, and the electroweak gauge bosons in the strongly interacting light Higgs (SILH) basis conventions, which can be written as $[34,35,42]$

$\mathcal{L}_{\text {eff }}=\mathcal{L}_{\mathrm{SM}}+\sum_{i} \bar{c}_{i} \mathcal{O}_{i} \equiv \mathcal{L}_{\mathrm{SM}}+\Delta \mathcal{L}_{F_{1}}+\Delta \mathcal{L}_{F_{2}}+\Delta \mathcal{L}_{\mathrm{SILH}}$,

where $\bar{c}_{i}$ coefficients are dimensionless Wilson coefficients, and $\mathcal{O}_{i}$ are dimension-six operators made up of SM fields. The first term in the effective Lagrangian of Eq. (2) is the SM Lagrangian, $\mathcal{L}_{\mathrm{SM}}$. The second term $\Delta \mathcal{L}_{F_{1}}$ in Eq. (2) addresses the interactions between two Higgs fields and a pair of quarks or leptons. This term has the following form:

$$
\begin{aligned}
\Delta \mathcal{L}_{F_{1}}= & \frac{i \bar{c}_{H Q}}{v^{2}}\left(\bar{q}_{L} \gamma^{\mu} q_{L}\right)\left(H^{\dagger} \stackrel{\leftrightarrow}{D}_{\mu} H\right)+\frac{i \bar{c}_{H Q}^{\prime}}{v^{2}}\left(\bar{q}_{L} \gamma^{\mu} \sigma^{i} q_{L}\right)\left(H^{\dagger} \sigma^{i} \stackrel{\leftrightarrow}{D}_{\mu} H\right) \\
& +\frac{i \bar{c}_{H u}}{v^{2}}\left(\bar{u}_{R} \gamma^{\mu} u_{R}\right)\left(H^{\dagger} \stackrel{\leftrightarrow}{D}_{\mu} H\right) \\
& +\frac{i \bar{c}_{H d}}{v^{2}}\left(\bar{d}_{R} \gamma^{\mu} d_{R}\right)\left(H^{\dagger} \stackrel{\leftrightarrow}{D}_{\mu} H\right)+\left[\frac{i \bar{c}_{H u d}}{v^{2}}\left(\bar{u}_{R} \gamma^{\mu} d_{R}\right)\left(H^{c^{\dagger}} \stackrel{\leftrightarrow}{D}_{\mu} H\right)+\text { H.c. }\right] \\
& +\frac{i \bar{c}_{H L}}{v^{2}}\left(\bar{L}_{L} \gamma^{\mu} L_{L}\right)\left(H^{\dagger} \stackrel{\leftrightarrow}{D}_{\mu} H\right) \\
& +\frac{i \bar{c}_{H L}^{\prime}}{v^{2}}\left(\bar{L}_{L} \gamma^{\mu} \sigma^{i} L_{L}\right)\left(H^{\dagger} \sigma^{i} \stackrel{\leftrightarrow}{D}_{\mu} H\right)+\frac{i \bar{c}_{H l}}{v^{2}}\left(\bar{l}_{R} \gamma^{\mu} l_{R}\right)\left(H^{\dagger} \stackrel{\leftrightarrow}{D}_{\mu} H\right) .
\end{aligned}
$$

The third term $\Delta \mathcal{L}_{F_{2}}$ of the effective Lagrangian in Eq. (2) contains the interactions of a pair of quarks or leptons, a Higgs field, and a gauge boson. This term reads

$$
\begin{aligned}
\Delta \mathcal{L}_{F_{2}}= & \frac{\bar{c}_{u B} g^{\prime}}{m_{W}^{2}} y_{u} \bar{q}_{L} H^{c} \sigma^{\mu \nu} u_{R} B_{\mu \nu}+\frac{\bar{c}_{u W} g}{m_{W}^{2}} y_{u} \bar{q}_{L} \sigma^{i} H^{c} \sigma^{\mu \nu} u_{R} W_{\mu \nu}^{i} \\
& +\frac{\bar{c}_{u G} g_{S}}{m_{W}^{2}} y_{u} \bar{q}_{L} H^{c} \sigma^{\mu \nu} \lambda^{a} u_{R} G_{\mu \nu}^{a}+\frac{\bar{c}_{d B} g^{\prime}}{m_{W}^{2}} y_{d} \bar{q}_{L} H \sigma^{\mu \nu} d_{R} B_{\mu \nu}+\frac{\bar{c}_{d W} g}{m_{W}^{2}} y_{d} \bar{q}_{L} \sigma^{i} H \sigma^{\mu \nu} d_{R} W_{\mu \nu}^{i} \\
& +\frac{\bar{c}_{d G} g_{S}}{m_{W}^{2}} y_{d} \bar{q}_{L} H \sigma^{\mu \nu} \lambda^{a} d_{R} G_{\mu \nu}^{a}+\frac{\bar{c}_{l B} g^{\prime}}{m_{W}^{2}} y_{l} \bar{L}_{L} H \sigma^{\mu \nu} l_{R} B_{\mu \nu} \\
& +\frac{\bar{c}_{l W} g}{m_{W}^{2}} y_{l} \bar{L}_{L} \sigma^{i} H \sigma^{\mu \nu} l_{R} W_{\mu \nu}^{i}+\text { H.c. }
\end{aligned}
$$


Finally, the last term of this Lagrangian corresponds to the Higgs field, which is part of a SILH. The $\Delta \mathcal{L}_{\text {SILH }}$ term can be expressed as

$$
\begin{aligned}
\Delta \mathcal{L}_{\mathrm{SILH}}= & \frac{\bar{c}_{H}}{2 v^{2}} \partial^{\mu}\left(H^{\dagger} H\right) \partial_{\mu}\left(H^{\dagger} H\right)+\frac{\bar{c}_{T}}{2 v^{2}}\left(H^{\dagger} \stackrel{\leftrightarrow}{ }^{\mu} H\right)\left(H^{\dagger} \stackrel{D}{D}_{\mu} H\right)-\frac{\bar{c}_{6} \lambda}{v^{2}}\left(H^{\dagger} H\right)^{3} \\
& +\left[\left[\frac{\bar{c}_{u}}{v^{2}} y_{u} H^{\dagger} H \bar{q}_{L} H^{c} u_{R}+\frac{\bar{c}_{d}}{v^{2}} y_{d} H^{\dagger} H \bar{q}_{L} H d_{R}+\frac{\bar{c}_{l}}{v^{2}} y_{l} H^{\dagger} H \bar{L}_{L} H l_{R}\right]+\text { H.c. }\right] \\
& +\frac{i \bar{c}_{W} g}{2 m_{W}^{2}}\left(H^{\dagger} \sigma^{i} \stackrel{\leftrightarrow}{D^{\mu}} H\right)\left(D^{\nu} W_{\mu \nu}\right)^{i} \\
& +\frac{i \bar{c}_{B} g \prime}{2 m_{W}^{2}}\left(H^{\dagger} \stackrel{\leftrightarrow}{\mu}^{\mu} H\right)\left(\partial^{\nu} B_{\mu \nu}\right)+\frac{i \bar{c}_{H W} g}{m_{W}^{2}}\left(D^{\mu} H\right)^{\dagger} \sigma^{i}\left(D^{\nu} H\right) W_{\mu \nu}^{i} \\
& +\frac{i \bar{c}_{H B} g^{\prime}}{m_{W}^{2}}\left(D^{\mu} H\right)^{\dagger}\left(D^{\nu} H\right) B_{\mu \nu}+\frac{\bar{c}_{\gamma} g^{2}}{m_{W}^{2}} H^{\dagger} H B_{\mu \nu} B^{\mu \nu}+\frac{\bar{c}_{g} g_{S}^{2}}{m_{W}^{2}} H^{\dagger} H G_{\mu \nu}^{a} G^{a \mu \nu} \\
& +\frac{i \tilde{c}_{H W} g}{m_{W}^{2}}\left(D^{\mu} H\right)^{\dagger} \sigma^{i}\left(D^{\nu} H\right) \tilde{W}_{\mu \nu}^{i}+\frac{i \tilde{c}_{H B} g^{\prime}}{m_{W}^{2}}\left(D^{\mu} H\right)^{\dagger}\left(D^{\nu} H\right) \tilde{B}_{\mu \nu} \\
& +\frac{\tilde{c}_{\gamma} g^{2}}{m_{W}^{2}} H^{\dagger} H B_{\mu \nu} \tilde{B}^{\mu \nu}+\frac{\tilde{c}_{g} g_{S}^{2}}{m_{W}^{2}} H^{\dagger} H G_{\mu \nu}^{a} \tilde{G}^{a \mu \nu} \\
& +\frac{\tilde{c}_{3 W} g^{3}}{m_{W}^{2}} \epsilon^{i j k} W_{\mu}^{i \nu} W_{\nu}^{j \rho} \tilde{W}_{\rho}^{k \mu}+\frac{\tilde{c}_{3 G} g_{S}^{3}}{m_{W}^{2}} f^{a b c} G_{\mu}^{a \nu} G_{\nu}^{b \rho} \tilde{G}_{\rho}^{c \mu}
\end{aligned}
$$

where $\Phi$ is a weak doublet containing the Higgs boson field, and $G^{\mu \nu}, B^{\mu \nu}, W^{\mu \nu}$ are the strong and electroweak field strength tensors. Here, $\Phi^{\dagger} \stackrel{\leftrightarrow}{D} \mu \Phi=\Phi^{\dagger}\left(D^{\mu} \Phi\right)-\left(D^{\mu} \Phi\right)^{\dagger} \Phi$ is the Hermitian covariant derivative. In Eq. (5), $\lambda$ is the Higgs boson quartic coupling and $v$ is the vacuum expectation value defined as $v=1 /\left(\sqrt{2} G_{F}\right)^{1 / 2}=246 \mathrm{GeV}$.

In the electron-proton colliders, the Higgs bosons are produced through two main channels: either via charged current $e^{-} q \rightarrow H q^{\prime} \nu_{e}$ or neutral current $e^{-} q \rightarrow e H q$. The leading order diagram for the production of a Higgs boson in the electron-proton collisions for the charged current process is depicted in Fig. 1. There have already been several studies on different aspects of the Higgs boson production via charged and neutral currents in the

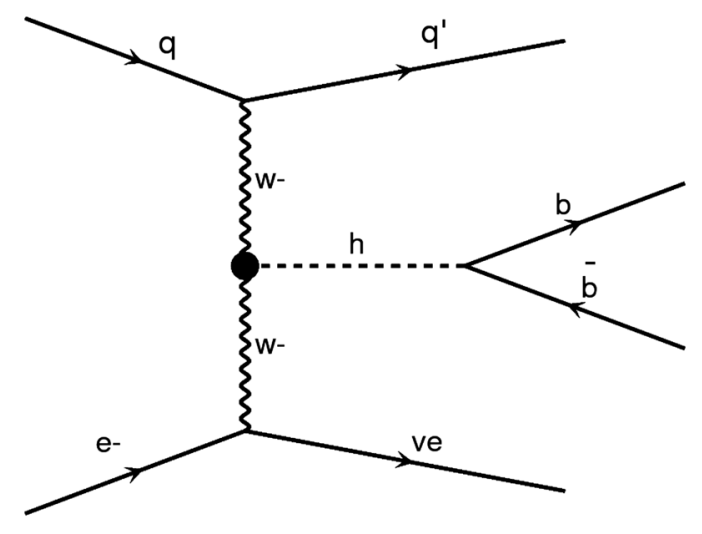

FIG. 1. Leading order Feynman diagram Higgs boson production via $e^{-} p \rightarrow h j \nu_{e}$ processes. electron-proton collisions [43-51]. In Ref. [47], it has been shown that the production cross section of the charged current process is larger than the neutral current process by a factor of a around 5 for the incoming electron energy of $140 \mathrm{GeV}$ and the proton energy of $7 \mathrm{TeV}$. In this work, our focus is on the charged current production process due to its larger production cross section. Also, this process has a clean signature as it is comprised of significant missing transverse energy and an energetic jet which tends to be forward. In this analysis, we concentrate on the Higgs boson decay into a pair of bottom quarks because of its large branching fraction.

The present work is dedicated to considering the effects of $\mathcal{L}_{\text {eff }}$ presented in Eq. (2) on the Higgs boson production through the charged current process $e^{-} q \rightarrow H q^{\prime} \nu_{e}$. The contributions originating from other possible effective operators are neglected for simplicity. The representative Feynman diagrams for $e^{-} q \rightarrow H q^{\prime} \nu_{e}$ are displayed in Fig. 2. The vertices that receive contributions from the $\mathcal{L}_{\text {eff }}$ are shown by filled circles. It is remarkable that the SM tree-level contribution does not have any dependence on the momenta of the involved particles; however, when considering $\mathcal{L}_{\text {eff }}$, momentum-dependent interactions enter the calculations. This leads to changes in the production cross sections as well as the shape of differential distributions. In this paper, differences in the shapes of distributions are used to constrain the involved Wilson coefficients in this process.

The $e^{-} p \rightarrow h j \nu_{e}$ process is sensitive to the following set of $\mathcal{L}_{\text {eff }}$ parameters: 
(a)

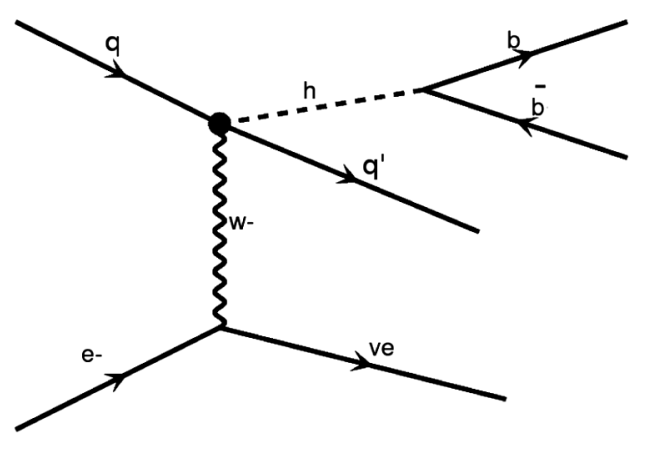

(b)

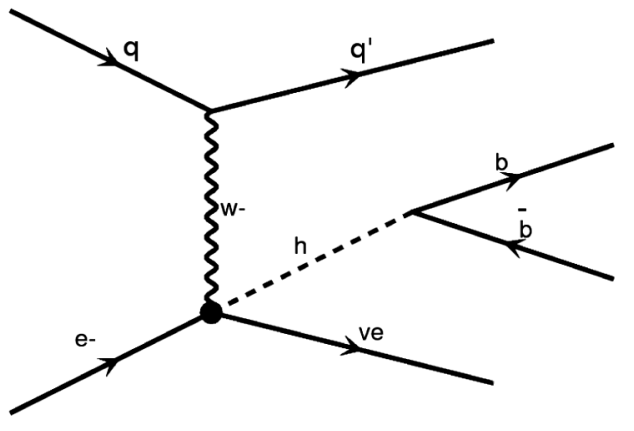

(c)

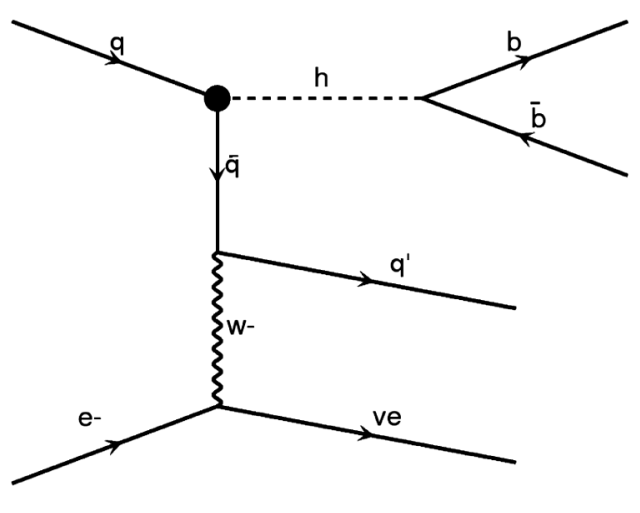

FIG. 2. Representative Feynman diagrams at tree level for the $e^{-} p \rightarrow h j \nu_{e}$ process in electron-proton collisions in the presence of dimension-six operators.

$\bar{c}_{H W}, \tilde{c}_{H W}, \bar{c}_{W}, \bar{c}_{H}, \bar{c}_{d}, \bar{c}_{u}, \bar{c}_{l}, \bar{c}_{H u d}, \bar{c}_{H L}^{\prime}, \bar{c}_{H Q}^{\prime}, \bar{c}_{u W}, \bar{c}_{d W}, \bar{c}_{e W}$.

The cross section of the $e^{-} p \rightarrow h j \nu_{e}$ process is found to be almost insensitive to the parameters $\bar{c}_{l}, \bar{c}_{e W}$, $\bar{c}_{d}, \bar{c}_{u}, \bar{c}_{u W}, \bar{c}_{d W}$. This is because of the very small Yukawa couplings of light quarks and electrons. As a result, our analysis is restricted to the remaining seven parameters:

$$
\bar{c}_{H}, \bar{c}_{H u d}, \bar{c}_{H W}, \bar{c}_{H L}^{\prime}, \bar{c}_{H Q}^{\prime}, \bar{c}_{W}, \quad \text { and } \tilde{c}_{H W} .
$$

An interesting way to represent the effective Lagrangian from the experimental and phenomenological points of

TABLE I. Anomalous Higgs boson couplings in the mass basis and their relation to the dimension-six coefficients.

\begin{tabular}{lc}
\hline \hline Mass basis & Gauge basis \\
\hline$g_{h w w}^{(1)}$ & $\frac{2 g}{m_{W}} \bar{c}_{H W}$ \\
$\tilde{g}_{h w w}$ & $\frac{2 g}{m_{W}} \tilde{c}_{H W}$ \\
$g_{\text {hww }}^{(2)}$ & $\frac{g}{m_{W}}\left\{\bar{c}_{W}+\bar{c}_{H W}\right\}$ \\
$g_{\text {hwud }}^{(L)}$ & $\frac{\sqrt{2} g}{v} \bar{c}_{H Q}^{\prime} V^{\mathrm{CKM}}$ \\
$g_{\text {hwud }}^{(R)}$ & $\frac{\sqrt{2} g}{v} \bar{c}_{H u d}$ \\
$g_{\text {hwue }}$ & $\frac{\sqrt{2} g}{v} \bar{c}_{H L}^{\prime}$ \\
\hline \hline
\end{tabular}

view is by the effective Lagrangian in the mass basis. In particular, it has been found to be an applicable approach in the study of electroweak precision tests (EWPT). The anomalous Higgs interactions in the mass basis have been presented in Ref. [35]. The relationship between the mass basis couplings and the dimension-six coefficients that are involved in this analysis are given in Table I.

There have already been many studies about constraining the Wilson coefficients discussed above in different colliders using various channels; these can be found in Refs. [7-25,52-54]. Although the obtained limits on some of the coefficients in the previous studies are tight, we examine possible improvements for these limits in the future high-energy electron-proton colliders via a careful investigation of the Higgs production mechanism in the framework of effective field theory. In the next section, the details of simulation for probing the effective Lagrangian using the $e^{-} p \rightarrow h j \nu_{e}$ process in the future LHeC and FCC-he colliders will be discussed.

\section{SIGNAL AND BACKGROUND PRODUCTION AND SIMULATION}

The chains we have used to perform the generation and simulation of the signal and background processes are described in this section. The full set of interactions generated by the dimension-six operators mentioned in the Higgs effective Lagrangian $\mathcal{L}_{\text {SILH }}$ of Eq. (5), $\Delta \mathcal{L}_{F_{1}}$ in 
Eq. (3), and $\Delta \mathcal{L}_{F_{2}}$ in Eq. (4) have been implemented in FeynRules [55,56], and the model is imported to a universal FeynRules output (UFO) module $[35,57]$. Then, the UFO model files are inserted into the MadGraph5-aMC@NLO $[58,59]$ Monte Carlo (MC) event generator to calculate the cross sections and generate the signal events. The CTEQ6L1 PDF set [60] is used to describe the proton structure functions. The renormalization and factorization scales are dynamical in MadGraph5-aMC@NLO.

The next-to-leading order QCD correction to the signal process $e^{-} p \rightarrow h j \nu_{e}$ is found to be small [61]. Therefore, in this work the $k$ factor for the signal is assumed to be 1 . The events of the signal process $e^{-} p \rightarrow h j \nu_{e}$ are generated with MadGraph5-aMC@NLO; then the Higgs boson decay into a $b \bar{b}$ pair via the MadSpin module $[62,63]$. The Pythia 6 $[64,65]$ package is utilized to perform fragmentation, hadronization, and initial- and final-state parton showers. Jets are clustered using FastJet3.2.0 [66] with the $k_{T}$ algorithm [67].

The electromagnetic and hadronic calorimeter resolutions are considered by the energy smearing of $\frac{5 \%}{\sqrt{E(\mathrm{GeV})}}$ (plus $1 \%$ of constant term) and $\frac{60 \%}{\sqrt{E(\mathrm{GeV})}}$, respectively [38]. The $b$-tagging efficiency is assumed to be $60 \%$, while mistag probabilities of $10 \%$ and $1 \%$ for $c$-quark jets and light-quark jets are considered, respectively [38].

The tracker of the $\mathrm{LHeC}$ detector is expected to cover a pseudorapidity range up to 3.0 [38]. Therefore, the $b$-tagging performance is valid up to $\left|\eta_{\text {b-jet }}\right|<3$. For the light jets, the calorimeter coverage is considered to be $\left|\eta_{\text {light-jet }}\right|<5$.

Based on the signal final state that consists of missing transverse energy, a pair of $b \bar{b}$ from the Higgs boson decay, and a forward jet, the backgrounds include processes with three jets and large missing energy in the final state. In particular, the following processes have been taken into account: $b b j^{\prime} \nu_{e}, b b b \nu_{e}, j^{\prime} j^{\prime} j^{\prime} \nu_{e}, t \nu_{e}, W j \nu_{e}$, and $Z j \nu_{e}$. The hadronic decays of the top quark, $W$ boson, and $Z$ boson are

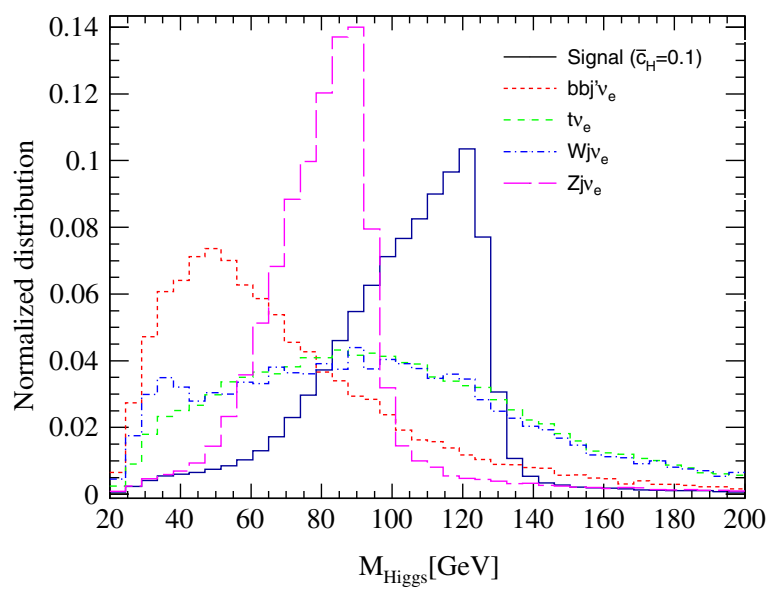

considered; $j^{\prime}$ refers to the light flavor jets except the $b$ quark, and $j$ denotes all light flavor quarks including the $b$ quark. The background contributions from photoproduction processes, which include the subprocesses $g \gamma \rightarrow b \bar{b}$ and $t \bar{t}$, are considered in the analysis.

In the next sections, we present the analysis strategies for LHeC and FCC-he separately in more detail. As mentioned before, we consider the $\mathrm{LHeC}$ with electron energies of $60 \mathrm{GeV}$ and $140 \mathrm{GeV}$ colliding with the $7 \mathrm{TeV}$ protons, while for the FCC-he case, the $60 \mathrm{GeV}$ electrons collide with $50 \mathrm{TeV}$ protons.

\section{LHeC SENSITIVITY}

In this section, we present the analysis strategy and the results for the $\mathrm{LHeC}$. The strategy for choosing the basic cuts is similar to the one proposed in [38]. Jets are reconstructed with a distance parameter for the jet reconstruction algorithm $R=0.7$. We require at least three jets with $p_{T}^{\text {jets }}>20 \mathrm{GeV}$, from which two are required to be $b$ tagged. A minimum cut of $20 \mathrm{GeV}$ is imposed on the missing transverse energy, and the total transverse energy of all final states needs to be greater than $100 \mathrm{GeV}$.

The Higgs boson is reconstructed using the two $b$-tagged jets that give the closest mass to the nominal Higgs mass, i.e., $125 \mathrm{GeV}$. Among the light jets, the highest $p_{T}$ one is taken as the light flavor jet. Figure 3 shows the reconstructed Higgs boson mass (left) and the invariant mass distribution of the Higgs + jet $\left(M_{\text {Higgs,j }}\right)$ (right) for signal with $\bar{c}_{H}=0.1$ and the main backgrounds after the preselection cuts.

In the reconstructed distribution of the Higgs boson mass, the mass peak is lower than the right Higgs boson mass because of the energy carried by the neutrino from the $b$-quark decays.

The cross sections (in fb) after each cut for the signal, SM production of Higgs boson via the $h j \nu_{e}$ process, and the main background processes are presented in Table II for

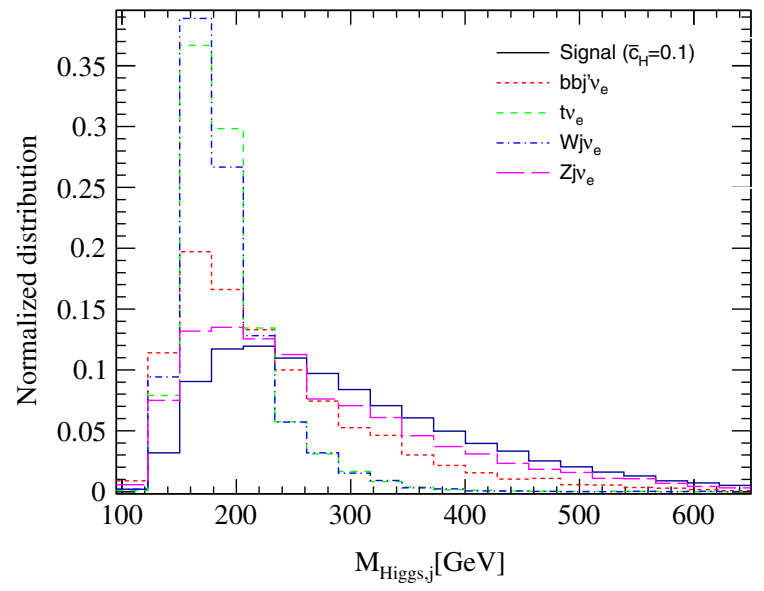

FIG. 3. The Higgs boson invariant mass distribution (left) and invariant mass distributions of Higgs + jet (right) after the basic cuts. 
TABLE II. Cross section (in fb) for signal and background events after applied kinematic cuts used for this analysis at the LHeC with $E_{e}=60 \mathrm{GeV}$. The details of the basic cuts applied are presented in the text.

\begin{tabular}{lccccrr}
\hline \hline \multirow{2}{*}{$\begin{array}{l}\text { LHeC collider } \\
\text { Cuts }\end{array}$} & \multirow{2}{*}{ Signal } & Standard model & \multicolumn{4}{c}{ Backgrounds } \\
\cline { 4 - 7 } & $\bar{c}_{H}=0.1$ & $h j \nu_{e}$ & $b b j^{\prime} \nu_{e}$ & $t \nu_{e}$ & $W j \nu_{e}$ & $Z j \nu_{e}$ \\
\hline Cross sections (in fb) & 84.8 & 94.3 & 639.5 & 1287 & 1885 & 379.6 \\
Acceptance cuts & 18.10 & 20.12 & 12.15 & 96.76 & 37.81 & 16.33 \\
$95 \leq M_{\text {Higgs }} \leq 135(\mathrm{GeV})$ & 9.69 & 13.07 & 1.28 & 23.60 & 10.08 & 1.52 \\
$260<M_{\text {Higgs,j }}<1000(\mathrm{GeV})$ & 6.37 & 7.05 & 0.45 & 1.77 & 0.76 & 0.72 \\
\hline \hline
\end{tabular}

the $\mathrm{LHeC}$ operating with an electron energy of $E_{e}=60 \mathrm{GeV}$. In addition to the basic kinematical cuts, a cut on the invariant mass of the Higgs boson $95 \leq M_{\text {Higgs }} \leq 135 \mathrm{GeV}$, as well as the invariant mass of the Higgs-jet system $260<M_{\text {Higgs,j }}<1000 \mathrm{GeV}$, is also applied for this analysis. The impact of these cuts is presented in Table II. It clearly shows that the selection criteria are effective in enhancing signal and suppression of the background contributions. As one can see, cuts on the Higgs boson invariant mass and the Higgs-jet system affect all backgrounds and reduce their contributions significantly. The cross section of the photoproduction background after all cuts is found to be $0.18 \mathrm{fb}$.

In this analysis, one might be concerned about the validity of the effective field theory. Several authors have discussed this issue in Refs. [68-70]. The Wilson coefficients of the dimension-six operators could be related to the new physics characteristic scale $M_{*}$ via

$$
\bar{c} \sim \frac{g_{*}^{2} v^{2}}{M_{*}^{2}},
$$

where $g_{*}$ is the coupling constant of the heavy degrees of freedom with the SM particles. Additional suppression factors appear in the case where an operator is generated at loop level. An upper bound can be put on the new mass scale $M_{*}$ using the fact that the underlying theory is strongly coupled by setting $g_{*}=4 \pi$. Assuming $\bar{c}=\mathcal{O}(1)$, we find

$$
M_{*}<\frac{4 \pi v}{\sqrt{\bar{c}}} \sim 3.2 \mathrm{TeV} .
$$

This upper bound is not violated in this analysis, as we have $M_{\text {Higgs,j }}<1 \mathrm{TeV}$.

\section{A. Sensitivity estimate}

This subsection is dedicated to estimating the sensitivity of the $e^{-} p \rightarrow h j \nu_{e}$ process to the Wilson coefficients. The sensitivity is obtained using a $\chi^{2}$ analysis over all bins of $\Delta_{E \mathrm{pz}}$ distribution. The $\Delta_{E \mathrm{pz}}$ variable is defined as

$$
\begin{aligned}
\Delta_{E \mathrm{pz}}= & \left(E_{b-\text { jet }_{1}}-p_{z, b-\text { jet }_{1}}\right)+\left(E_{b-\text { jet }_{2}}-p_{z, b-\text { jet }_{2}}\right) \\
& +\left(E_{\text {light-jet }_{1}}-p_{z, \text { light-jet }_{1}}\right) .
\end{aligned}
$$

In Fig. 4, we show the expected normalized distribution of $\Delta_{E \mathrm{pz}}$ for the signal and the main sources of background processes after applying all cuts presented in Table II. As we can see, the shape of the $\Delta_{E \mathrm{pz}}$ signal with $\bar{c}_{H}=0.1$ is quite different from the sum of all background processes. As a result, it is useful to obtain the exclusion limits on the Wilson coefficients defined in Eqs. (3) and (5).

To set upper limits at the $95 \%$ CL, we use a $\chi^{2}$ criterion from the distribution of $\Delta_{E \mathrm{pz}}$ defined as

$$
\chi^{2}\left(\left\{c_{n}\right\}\right)=\sum_{i=\text { bins }}^{N} \frac{\left(f_{i}\left(\left\{c_{n}\right\}\right)-s_{i}^{\mathrm{SM}}\right)^{2}}{\Delta_{i}^{2}},
$$

where $\left\{c_{n}\right\}$ denotes the Wilson coefficient $\left\{c_{n}=\bar{c}_{H}, \bar{c}_{H u d}\right.$, $\left.\bar{c}_{H W}, \bar{c}_{H L}^{\prime}, \bar{c}_{H Q}^{\prime}, \bar{c}_{W}, \tilde{c}_{H W}\right\}$ considered in the present analysis, $s_{i}^{\mathrm{SM}}$ refers to the SM expectation in the $i$ th bin of the $\Delta_{E \mathrm{pz}}$ distribution, and $f_{i}\left(\left\{c_{n}\right\}\right)$ is the number of signal events in the $i$ th bin. In the $\chi^{2}\left(\left\{c_{n}\right\}\right)$ definition, $\Delta_{i}$ is the statistical uncertainty. We consider the most general formulation of $f_{i}\left(\left\{c_{n}\right\}\right)$ as second-degree polynomials according to the following:

$$
f_{i}\left(\left\{c_{n}\right\}\right)=s_{i}^{\mathrm{SM}}+\sum_{n=1}^{N}\left(\alpha_{n} \bar{c}_{n}+\beta_{n} \bar{c}_{n}^{2}\right) .
$$

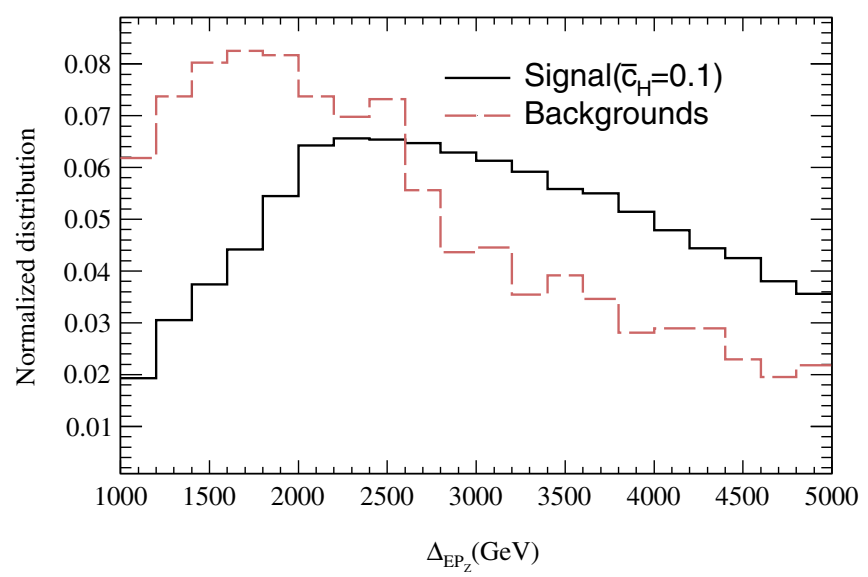

FIG. 4. Normalized distribution for $\Delta_{E \mathrm{pz}}$ for signal and all background processes after applying all cuts presented in Table II. 
TABLE III. Predicted constraints at $95 \% \mathrm{CL}$ on dimension-six Wilson coefficients for the LHeC with electrons energies of $E_{e}=$ $60 \mathrm{GeV}$ and $E_{e}=140 \mathrm{GeV}$, and for integrated luminosities of $300 \mathrm{fb}^{-1}$ and $3000 \mathrm{fb}^{-1}$.

\begin{tabular}{|c|c|c|c|c|c|}
\hline $\begin{array}{l}\text { Wilson } \\
\text { coefficients }\end{array}$ & $\begin{array}{c}\text { LHeC-300 } \\
\left(E_{e}=140 \mathrm{GeV}\right)\end{array}$ & $\begin{array}{c}\text { LHeC-3000 } \\
\left(E_{e}=140 \mathrm{GeV}\right)\end{array}$ & $\begin{array}{c}\text { LHeC-300 } \\
\left(E_{e}=60 \mathrm{GeV}\right)\end{array}$ & $\begin{array}{c}\text { LHeC-3000 } \\
\left(E_{e}=60 \mathrm{GeV}\right)\end{array}$ & $\begin{array}{c}\text { LHC-3000 } \\
\text { [20] }\end{array}$ \\
\hline $\bar{c}_{H}[\times 100]$ & {$[-0.90,0.95]$} & {$[-0.29,0.29]$} & {$[-7.8,8.8]$} & {$[-2.5,2.6]$} & {$[-4.40,3.50]$} \\
\hline $\bar{c}_{H u d}[\times 100]$ & {$[-0.80,0.80]$} & {$[-0.25,0.25]$} & {$[-6.26,8.33]$} & {$[-2.40,2.86]$} & $\ldots$ \\
\hline $\bar{c}_{H W}[\times 100]$ & {$[-1.40,1.70]$} & {$[-0.47,0.50]$} & {$[-2.3,2.8]$} & {$[-0.79,0.83]$} & {$[-0.4,0.4]$} \\
\hline $\bar{c}_{H L}^{\prime}[\times 100]$ & {$[-1.30,1.40]$} & {$[-0.40,0.40]$} & {$[-2.6,2.7]$} & {$[-0.85,0.82]$} & $\ldots$ \\
\hline $\bar{c}_{H Q}^{\prime}[\times 100]$ & {$[-1.50,1.60]$} & {$[-0.50,0.50]$} & {$[-2.20,2.70]$} & {$[-0.79,0.76]$} & $\ldots$ \\
\hline $\bar{c}_{W}[\times 100]$ & {$[-1.00,1.00]$} & {$[-0.36,0.37]$} & {$[-1.20,1.40]$} & {$[-0.42,0.44]$} & {$[-0.40,0.40]$} \\
\hline$\tilde{c}_{H W}[\times 100]$ & {$[-0.70,0.70]$} & {$[-0.20,0.20]$} & {$[-11.4,9.2]$} & {$[-4.2,3.6]$} & $\ldots$ \\
\hline
\end{tabular}

Considering only one coefficient in the fit, one can obtain exclusion limits for the individual constraints on the Wilson coefficient $\left\{c_{n}=\bar{c}_{H}, \bar{c}_{H u d}, \bar{c}_{H W}, \bar{c}_{H L}^{\prime}, \bar{c}_{H Q}^{\prime}\right.$, $\left.\bar{c}_{W}, \tilde{c}_{H W}\right\}$. The corresponding results for the integrated luminosities of $300 \mathrm{fb}^{-1}, 3000 \mathrm{fb}^{-1}$, and $1 \mathrm{ab}^{-1}$ are presented in Tables III and V with electron energies of $60 \mathrm{GeV}$ and $140 \mathrm{GeV}$. As an example, $\mathrm{LHeC}$ would be able to constrain $\bar{c}_{H}$ by more than 1 order of magnitude with respect to the LHC in the high-luminosity regime.

\section{FCC-HE SENSITIVITY}

In this section, the sensitivity of the FCC-he to the related Wilson coefficients in the $h j \nu_{e}$ process is studied. As we mentioned before, FCC-he employs a $50 \mathrm{TeV}$ proton beam of a proposed circular proton-proton collider.

Similar to the LHeC case, FCC-he is sensitive to $\bar{c}_{H}, \bar{c}_{H u d}, \bar{c}_{H W}, \bar{c}_{H L}^{\prime}, \bar{c}_{H Q}^{\prime}, \bar{c}_{W}$, and $\tilde{c}_{H W}$ Wilson coefficients. The same analysis strategy as presented for the $\mathrm{LHeC}$ is followed for the FCC-he. The Higgs boson decay into a $b \bar{b}$ pair is considered, and a $\chi^{2}$ fit is performed to estimate the sensitivities. The ratio of the cross section of $e^{-} p \rightarrow h j \nu_{e}$ at the FCC-he to the LHeC in terms of the Wilson coefficients is presented in Fig. 5. As we can see, when the couplings vary in the range -0.03 to 0.03 , the cross section at the FCC-he increases by a factor of around 6 with respect to the $\mathrm{LHeC}$.

At this step, we mention one of the interesting characteristics of the signal events at the FCC-he, which requires using a particular strategy for reconstruction of the Higgs boson. At the FCC-he, Higgs bosons for some scenarios of the signal are highly boosted, and from the topological point of view, they decay differently compared to the Higgs bosons which are not boosted. For instance, Higgs bosons produced in the signal scenario with a nonzero value of $\bar{c}_{H W}$ are highly boosted because of the momentum-dependent interaction. For the splitting of the Higgs boson into a pair of $b \bar{b}$, one can write

$$
m_{H}^{2} \simeq 2\left|\vec{p}_{b}\right|\left|\vec{p}_{\bar{b}}\right|\left(1-\cos \theta_{b \bar{b}}\right),
$$

where $\vec{p}_{b}\left(\vec{p}_{\bar{b}}\right)$ is the momentum of the $b(\bar{b})$ quark and the bottom quark mass has been neglected. One can express the opening angle $\theta_{b \bar{b}}$ versus the parent mass Higgs boson and the momenta of the $b$ and $\bar{b}$ quarks. Using kinematic relations, the angular separation of a $b \bar{b}$ pair produced in a Higgs boson decay can also be written as

$$
\Delta R_{b \bar{b}} \simeq \frac{1}{\sqrt{x(1-x)}} \frac{m_{H}}{p_{T}},
$$

where $p_{T}$ is the transverse momentum of the Higgs boson, and $x$ and $1-x$ are the momentum fractions of the $b$ and $\bar{b}$ quarks. Figure 6 shows the distributions of the Higgs boson momentum and transverse momentum for the signal scenario of $\bar{c}_{H W}=0.1$ for the LHeC and FCC-he. As we can see, at the FCC-he, Higgs bosons reside at large values of momentum and $p_{T}$.

For the Higgs bosons with substantial momentum and $p_{T}$, from Eq. (12) and (13) it is expected that the angular separation of the Higgs boson decay products decreases. Figure 7 shows the normalized distribution of $\Delta R$ between two $b$ quarks from the Higgs boson decay for the FCC-he. We present the distributions for two signal scenarios $\bar{c}_{H}=$ 0.1 and $\bar{c}_{H W}=0.1$. The plot clearly confirms that for the

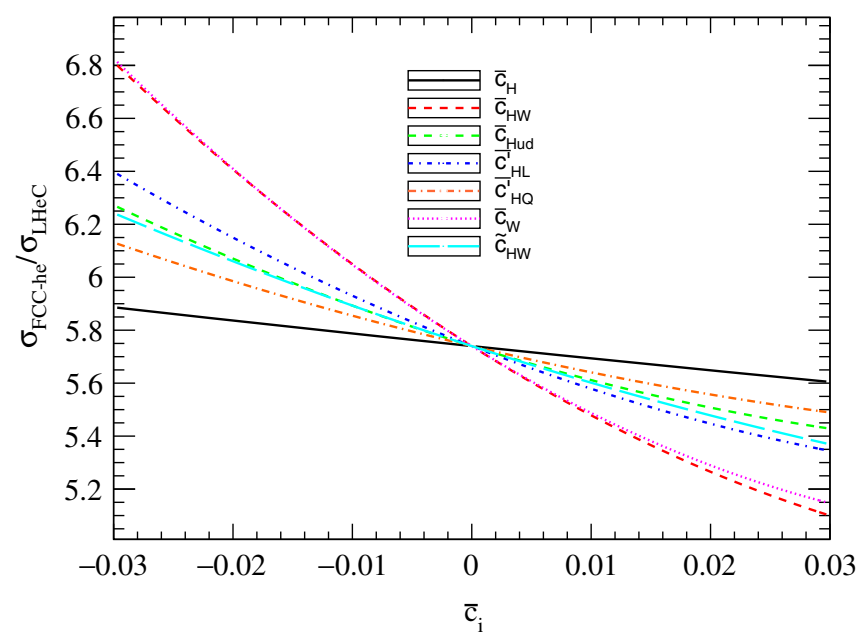

FIG. 5. The ratio of the signal cross section at the FCC-he $\left(E_{e}=60 \mathrm{GeV}\right.$ and $\left.E_{p}=50 \mathrm{TeV}\right)$ to the LHeC $\left(E_{e}=60 \mathrm{GeV}\right.$ and $E_{p}=7 \mathrm{TeV}$ ) versus various Wilson coefficients. 

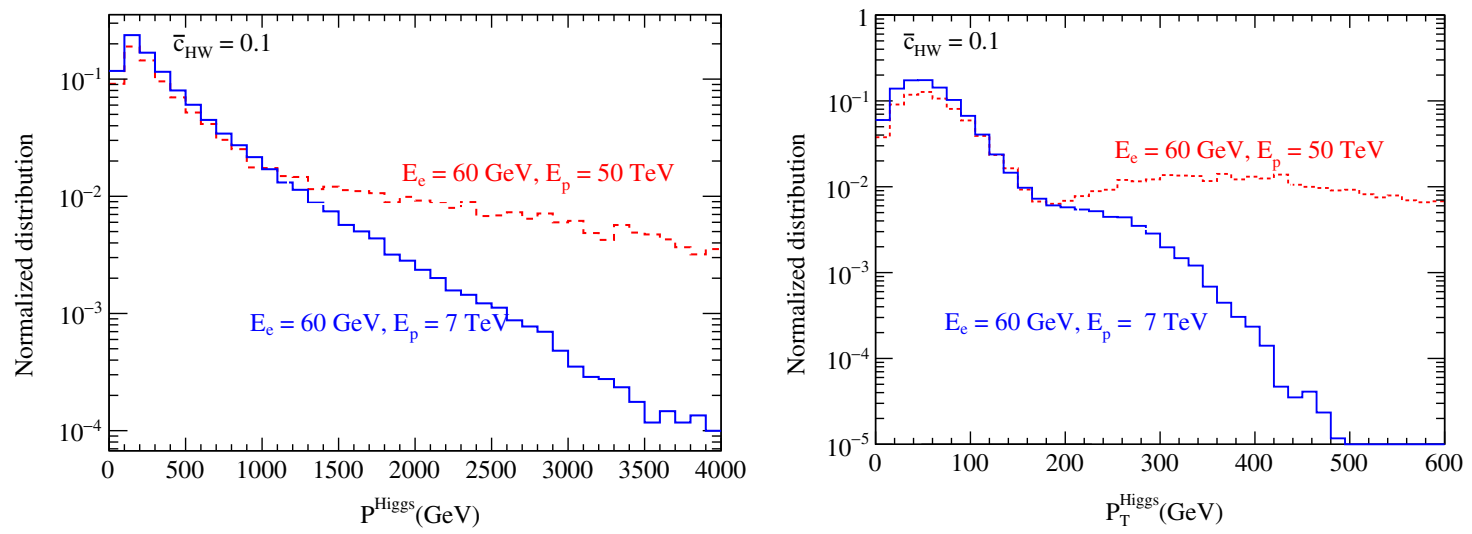

FIG. 6. The distributions of the Higgs boson momentum (left) and the Higgs boson transverse momentum (right) at the LHeC and FCC-he for the signal scenario of $\bar{c}_{H W}=0.1$.

signal scenario of $\bar{c}_{H W}$, a considerable fraction of Higgs bosons are produced in the boosted regime, while this is not valid for the signal scenario of $\bar{c}_{H}$. As a result, the high- $p_{T}$ Higgs bosons produce a collimated jet with substructure.

Because of the small angular separation between two $b$ jets from the Higgs decay and large boost, the common jet reconstruction with a cone size of $\Delta R=0.4-0.5$ would not be usable for most of the signal events with a nonzero value of $\bar{c}_{H W}$. An alternative method of the fat jet algorithm is applied [71] for these boosted events.

To reconstruct the signal events with two boosted $b$ jets in the final state, first we reconstruct the fat jets by using the Cambridge/Aachen (CA) jet algorithm [72,73] assuming a jet cone size of $R=1.2$. Then, in order to identify the boosted Higgs boson, the methods described in the fat jet reconstruction algorithm [71] are explained in the following. In the beginning, a reconstructed fat jet $J$ is split into two subjets $J_{1}, J_{2}$ with masses $m_{J_{1}}>m_{J_{2}}$. Then, the method requires a significant mass drop of $m_{J_{1}}<$ $\mu_{\mathrm{MD}} m_{J}$ with $\mu=0.667$. It should be mentioned that $\mu_{\mathrm{MD}}$ is

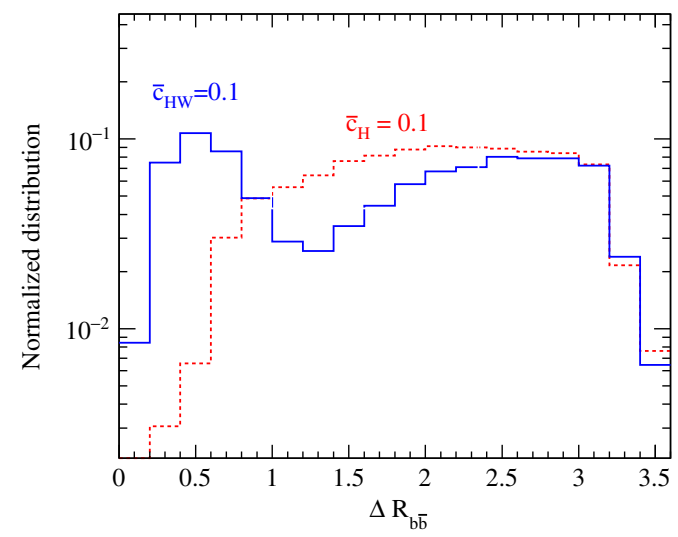

FIG. 7. Normalized distribution of $\Delta R$ between two $b$ quarks coming from the decay of Higgs bosons for two signal scenarios of $\bar{c}_{H}=0.1$ and $\bar{c}_{H W}=0.1$ at the FCC-he. an arbitrary parameter that shows the degree of mass drop. Also, to avoid including high- $p_{T}$ light jets, two subjets need to be symmetrically split. Thus, the two subjets must satisfy

$$
\frac{\min \left(p_{T, J_{1}}^{2}, p_{T, J_{2}}^{2}\right)}{m_{J}^{2}} \Delta R_{J_{1}, J_{2}}^{2}<y_{\text {cut }}
$$

where $y_{\text {cut }}$ is a parameter of the algorithm which determines the limit of asymmetry between two subjets, and $p_{T, J_{1}}^{2}$ and $p_{T, J_{2}}^{2}$ are the squares of the transverse momentum of each subjet. Finally, if the above criteria are not satisfied, the algorithm takes $J=J_{1}$ and returns to the first step for performing decomposition. The explained algorithm for boosted object reconstruction has been implemented in the FastJet package [66] by which our analysis is done.

In this analysis, jets of both the signal and background are first reconstructed with the $k_{T}$ algorithm with a cone size of $R=0.7$. Then, if jets with $p_{T}>250 \mathrm{GeV}$ are found, the fat jet algorithm is applied; otherwise, the event is considered to be a normal event. The exclusion limits for the Wilson coefficients of the dimension-six operators at the FCC-he are obtained using a method similar to what was described in Sec. IVA.

\section{RESULTS}

In this section, the results are presented for the electronproton collisions at the $\mathrm{LHeC}$ when the $60 \mathrm{GeV}$ and $140 \mathrm{GeV}$ electrons collide with the $7 \mathrm{TeV}$ protons, and at the FCC-he when the $60 \mathrm{GeV}$ electrons collide with the $50 \mathrm{TeV}$ protons. The limits are presented for the integrated luminosities of $300 \mathrm{fb}^{-1}, 3000 \mathrm{fb}^{-1}$, and $1 \mathrm{ab}^{-1}$ in Tables III-V.

In Tables III and IV, we present the constraints on the Wilson coefficients that have been obtained at the LHC at $14 \mathrm{TeV}$ with an integrated luminosity of $3000 \mathrm{fb}^{-1}$ [20]. As we can see from these tables, more sensitivity is 
TABLE IV. Predicted constraints at $95 \% \mathrm{CL}$ on dimension-six Wilson coefficients for the LHeC and FCC-he colliders and for integrated luminosity of $300 \mathrm{fb}^{-1}$ and $3000 \mathrm{fb}^{-1}$.

\begin{tabular}{|c|c|c|c|c|c|}
\hline $\begin{array}{l}\text { Wilson } \\
\text { coefficients }\end{array}$ & $\begin{array}{c}\text { LHeC-300 } \\
\left(E_{e}=60 \mathrm{GeV}\right)\end{array}$ & $\begin{array}{c}\text { LHeC-3000 } \\
\left(E_{e}=60 \mathrm{GeV}\right)\end{array}$ & $\begin{array}{c}\text { FCC-he-300 } \\
\left(E_{e}=60 \mathrm{GeV}\right)\end{array}$ & $\begin{array}{l}\text { FCC-he-3000 } \\
\left(E_{e}=60 \mathrm{GeV}\right)\end{array}$ & $\begin{array}{c}\mathrm{LHC}-3000 \\
{[20]}\end{array}$ \\
\hline $\bar{c}_{H}[\times 100]$ & {$[-7.8,8.8]$} & {$[-2.5,2.6]$} & {$[-8.70,8.70]$} & {$[-2.75,2.75]$} & {$[-4.40,3.50]$} \\
\hline $\bar{c}_{\text {Hud }}[\times 100]$ & {$[-6.26,8.33]$} & {$[-2.40,2.86]$} & {$[-4.00,4.00]$} & {$[-1.26,1.26]$} & $\ldots$ \\
\hline $\bar{c}_{H W}[\times 100]$ & {$[-2.3,2.8]$} & {$[-0.79,0.83]$} & {$[-1.00,1.10]$} & {$[-0.32,0.35]$} & {$[-0.4,0.4]$} \\
\hline $\bar{c}_{H L}^{\prime}[\times 100]$ & {$[-2.6,2.7]$} & {$[-0.85,0.82]$} & {$[-4.50,4.90]$} & {$[-1.42,1.54]$} & $\ldots$ \\
\hline $\bar{c}_{H Q}^{\prime}[\times 100]$ & {$[-2.20,2.70]$} & {$[-0.79,0.76]$} & {$[-5.70,6.00]$} & {$[-1.80,1.90]$} & $\cdots$ \\
\hline $\bar{c}_{W}[\times 100]$ & {$[-1.20,1.40]$} & {$[-0.42,0.44]$} & {$[-1.20,1.30]$} & {$[-0.38,0.41]$} & {$[-0.40,0.40]$} \\
\hline$\tilde{c}_{H W}[\times 100]$ & {$[-11.4,9.2]$} & {$[-4.2,3.6]$} & {$[-4.70,4.70]$} & {$[-1.49,1.49]$} & $\ldots$ \\
\hline
\end{tabular}

achievable on the coefficient $\bar{c}_{H}$ at the electron-proton colliders with respect to the LHC. Comparison between LHeC and FCC-he sensitivities shows that more sensitivity to most of the Wilson coefficients can be obtained in the FCC-he. From these results, one can conclude that the LHeC and FCC-he are suitable platforms to complement the LHC results in the search for dimension-six effective couplings in the Higgs boson sector.

In order to study the effect arising from different energies of colliding electrons, we present the results for the LHeC with the electron energies of $E_{e}=60 \mathrm{GeV}$ and $E_{e}=140 \mathrm{GeV}$. From Table III, it can be seen that going to higher energies of the electron-proton collisions, from $60 \mathrm{GeV}$ to $140 \mathrm{GeV}$, would lead to improvements for the Wilson coefficients. For example, the constraints obtained from $E_{e}=60 \mathrm{GeV}$ with the integrated luminosity of $3000 \mathrm{fb}^{-1}$ on $\bar{c}_{H}$ are $-0.025<\bar{c}_{H}<0.026$, which is tightened to $-0.0029<\bar{c}_{H}<0.0029$ at a $E_{e}=140 \mathrm{GeV}$ machine.

The results for the $\mathrm{LHeC}$ and FCC-he at very high integrated luminosities are presented in Table V. The bounds are given for maximum achievable integrated luminosities of $1 \mathrm{ab}^{-1}$ and $10 \mathrm{ab}^{-1}$ for the LHeC and FCC-he, respectively. Based on this analysis for the FCChe with $E_{e}=60 \mathrm{GeV}$ for an integrated luminosity of

TABLE V. Predicted constraints at $95 \% \mathrm{CL}$ on Wilson coefficients for the LHeC and FCC-he with $E_{e}=60 \mathrm{GeV}$. The limits are presented for the integrated luminosities of $1 \mathrm{ab}^{-1}$ for the LHeC and $10 \mathrm{ab}^{-1}$ for the FCC-he, respectively.

\begin{tabular}{lcc}
\hline \hline $\begin{array}{l}\text { Wilson } \\
\text { coefficients }\end{array}$ & $\begin{array}{c}\text { LHeC } \\
\left(E_{e}=60 \mathrm{GeV}\right) 1 \mathrm{ab}^{-1}\end{array}$ & $\begin{array}{c}\text { FCC-he } \\
\left(E_{e}=60 \mathrm{GeV}\right) 10 \mathrm{ab}^{-1}\end{array}$ \\
\hline $\bar{c}_{H}[\times 100]$ & {$[-4.28,4.60]$} & {$[-1.5,1.5]$} \\
$\bar{c}_{H u d}[\times 100]$ & {$[-3.88,4.96]$} & {$[-0.69,0.69]$} \\
$\bar{c}_{H W}[\times 100]$ & {$[-0.89,0.96]$} & {$[-0.17,0.19]$} \\
$\bar{c}_{H L}^{\prime}[\times 100]$ & {$[-1.43,1.58]$} & {$[-0.78,0.85]$} \\
$\bar{c}_{H Q}^{\prime}[\times 100]$ & {$[-1.29,1.4]$} & {$[-0.98,1.01]$} \\
$\bar{c}_{W}[\times 100]$ & {$[-0.71,0.76]$} & {$[-0.21,0.22]$} \\
$\tilde{c}_{H W}[\times 100]$ & {$[-7.05,6.03]$} & {$[-0.81,0.81]$} \\
\hline \hline
\end{tabular}

$10 \mathrm{ab}^{-1}$, the sensitivity to the Wilson coefficients is much better than the other options analyzed in this study, and in some cases, it is better than the ones expected to be achieved by the HL-LHC with an integrated luminosity of $3000 \mathrm{fb}^{-1}$.

\section{SUMMARY AND CONCLUSIONS}

The effects of physics beyond the SM may appear in the Higgs sector, which requires one to measure the Higgs boson couplings with the SM particles precisely. Any deviation of the Higgs boson interactions with respect to the predictions of the SM would be a hint to new physics. The $\mathrm{LHeC}$ with a rich physics program would be able to provide a lot of information on physics beyond the SM as well as precise measurements of the SM. In electron-proton collisions, there are two clean production mechanisms for the Higgs boson, either in neutral current interactions or in charged current interactions. In this paper, we present an analysis to constrain new physics in the Higgs boson sector by adopting an effective Lagrangian approach. The analysis is based on Higgs boson production in charged current interactions (via the $W^{+} W^{-} H$ coupling), i.e., the $e^{-} p \rightarrow h j \nu_{e}$ process for the electrons with energies of $E_{e}=60 \mathrm{GeV}$ and $E_{e}=$ $140 \mathrm{GeV}$ colliding with the $7 \mathrm{TeV}$ protons. We also perform the same analysis for the FCC-he in which $60 \mathrm{GeV}$ electrons collide with very high-energy protons, with energy of $50 \mathrm{TeV}$. For the FCC-he case, to efficiently reconstruct the Higgs boson and to achieve a reasonable background rejection, jet substructure techniques are used in order to capture the signal events that are boosted objects.

To obtain the sensitivity to the involved Wilson coefficients of dimension-six operators, an analysis on the kinematic distribution of $\Delta_{E \mathrm{pz}}$ [defined in Eq. (9)] is performed. The Higgs boson production via charged current interaction, $e^{-} p \rightarrow h j \nu_{e}$, is, in particular, sensitive to a variety of Wilson coefficients, namely, $\left\{c_{i}=\bar{c}_{H}\right.$, $\left.\bar{c}_{H u d}, \bar{c}_{H W}, \bar{c}_{H L}^{\prime}, \bar{c}_{H Q}^{\prime}, \bar{c}_{W}, \tilde{c}_{H W}\right\}$. The extracted bounds for both the LHeC and FCC-he, which are presented in 
Table IV, show a great sensitivity, and in some cases, improvements are expected with respect to the potential constraints for the LHC $[20,23]$. We also show that the FCC-he collider with $E_{e}=60 \mathrm{GeV}$ and with an integrated luminosity of $\mathcal{L}=10 \mathrm{ab}^{-1}$ or even with $3 \mathrm{ab}^{-1}$ would be able to probe the Wilson coefficients of dimension-six operators of the Higgs boson (especially $\bar{c}_{H}, \bar{c}_{H W}$, and $\bar{c}_{W}$ couplings) beyond the HL-LHC.

\section{ACKNOWLEDGMENTS}

The authors thank the School of Particles and Accelerators, Institute for Research in Fundamental Sciences (IPM) for financial support of this project. H. K. is also grateful to the University of Science and Technology of Mazandaran for financial support provided for this research. M. M. N. acknowledges the Iran National Science Foundation (INSF) for support.
[1] G. Aad et al. (ATLAS Collaboration), Observation of a new particle in the search for the Standard Model Higgs boson with the ATLAS detector at the LHC, Phys. Lett. B 716, 1 (2012).

[2] S. Chatrchyan et al. (CMS Collaboration), Observation of a new boson at a mass of $125 \mathrm{GeV}$ with the CMS experiment at the LHC, Phys. Lett. B 716, 30 (2012).

[3] W. Buchmuller and D. Wyler, Effective Lagrangian analysis of new interactions and flavor conservation, Nucl. Phys. B268, 621 (1986).

[4] B. Grzadkowski, M. Iskrzynski, M. Misiak, and J. Rosiek, Dimension-six terms in the standard model Lagrangian, J. High Energy Phys. 10 (2010) 085.

[5] J. A. Aguilar-Saavedra, A minimal set of top anomalous couplings, Nucl. Phys. B812, 181 (2009).

[6] C. Arzt, M. B. Einhorn, and J. Wudka, Patterns of deviation from the standard model, Nucl. Phys. B433, 41 (1995).

[7] C. Hartmann, W. Shepherd, and M. Trott, The $Z$ decay width in the SMEFT: $y_{t}$ and $\lambda$ corrections at one loop, J. High Energy Phys. 03 (2017) 060.

[8] S. Kuday, H. Saygin, I. Hos, and F. Cetin, Projections for neutral Di-Boson and Di-Higgs interactions at FCC-he collider, arXiv:1702.00185.

[9] W. Kilian, S. Sun, Q. S. Yan, X. Zhao, and Z. Zhao, New physics in multi-Higgs boson final states, J. High Energy Phys. 06 (2017) 145.

[10] J. Ellis, P. Roloff, V. Sanz, and T. You, Dimension-6 operator analysis of the CLIC sensitivity to new physics, J. High Energy Phys. 05 (2017) 096.

[11] S. Fichet, A. Tonero, and P. R. Teles, Sharpening the shape analysis for higher-dimensional operator searches, Phys. Rev. D 96, 036003 (2017).

[12] C. Sigismondi, Measuring the position of the center of the Sun at the Clementine Gnomon of Santa Maria degli Angeli in Rome, J. Occult. Astron. 1N5, 20 (2012).

[13] A. Arbey, S. Fichet, F. Mahmoudi, and G. Moreau, The correlation matrix of Higgs rates at the LHC, J. High Energy Phys. 11 (2016) 097.

[14] G. Amar, S. Banerjee, S. von Buddenbrock, A. S. Cornell, T. Mandal, B. Mellado, and B. Mukhopadhyaya, Exploration of the tensor structure of the Higgs boson coupling to weak bosons in $e^{+} e^{-}$collisions, J. High Energy Phys. 02 (2015) 128.
[15] S. Banerjee, T. Mandal, B. Mellado, and B. Mukhopadhyaya, Cornering dimension-6 $H V V$ interactions at high luminosity LHC: The role of event ratios, J. High Energy Phys. 09 (2015) 057.

[16] N. Craig, J. Gu, Z. Liu, and K. Wang, Beyond Higgs couplings: Probing the Higgs with angular observables at future $e^{+} e^{-}$colliders, J. High Energy Phys. 03 (2016) 050 .

[17] T. Corbett, O. J. P. Eboli, D. Goncalves, J. Gonzalez-Fraile, T. Plehn, and M. Rauch, The Higgs legacy of the LHC run I, J. High Energy Phys. 08 (2015) 156.

[18] J. Ellis, V. Sanz, and T. You, The effective standard model after LHC run I, J. High Energy Phys. 03 (2015) 157.

[19] L. Berthier and M. Trott, Consistent constraints on the standard model effective field theory, J. High Energy Phys. 02 (2016) 069.

[20] C. Englert, R. Kogler, H. Schulz, and M. Spannowsky, Higgs coupling measurements at the LHC, Eur. Phys. J. C 76, 393 (2016)

[21] J. Ellis, V. Sanz, and T. You, Complete Higgs sector constraints on dimension-6 operators, J. High Energy Phys. 07 (2014) 036.

[22] H. Khanpour and M. M. Najafabadi, Constraining Higgs boson effective couplings at electron-positron colliders, Phys. Rev. D 95, 055026 (2017).

[23] H. Khanpour, S. Khatibi, and M. M. Najafabadi, Probing Higgs boson couplings in $\mathrm{H}+\gamma$ production at the LHC, Phys. Lett. B 773, 462 (2017).

[24] A. Buckley, C. Englert, J. Ferrando, D. J. Miller, L. Moore, M. Russell, and C.D. White, Constraining top quark effective theory in the LHC run II era, J. High Energy Phys. 04 (2016) 015.

[25] A. Buckley, C. Englert, J. Ferrando, D. J. Miller, L. Moore, M. Russell, and C.D. White, Global fit of top quark effective theory to data, Phys. Rev. D 92, 091501 (2015).

[26] H. Denizli and A. Senol, Constraints on Higgs effective couplings in $H \nu \bar{\nu}$ production of CLIC at $380 \mathrm{GeV}$, Adv. High Energy Phys. 2018, 1627051 (2018).

[27] T. Barklow, K. Fujii, S. Jung, R. Karl, J. List, T. Ogawa, M. E. Peskin, and J. Tian, Improved formalism for precision Higgs coupling fits, Phys. Rev. D 97, 053003 (2018).

[28] S. Jana and S. Nandi, New Physics Scale from Higgs Observables with Effective Dimension-6 Operators, arXiv:1710.00619. 
[29] A. Dedes, W. Materkowska, M. Paraskevas, J. Rosiek, and K. Suxho, Feynman rules for the Standard Model Effective Field Theory in $R_{\xi}$ - gauges, J. High Energy Phys. 06 (2017) 143.

[30] A. Dedes, M. Paraskevas, J. Rosiek, K. Suxho, and L. Trifyllis, The decay $h \rightarrow \gamma \gamma$ in the Standard-Model Effective Field Theory, arXiv:1805.00302.

[31] S. Bar-Shalom and A. Soni, A universally enhanced lightquarks Yukawa couplings paradigm, arXiv:1804.02400.

[32] J. Gu, H. Li, Z. Liu, S. Su, and W. Su, Learning from Higgs Physics at Future Higgs Factories, J. High Energy Phys. 12 (2017) 153.

[33] C. W. Murphy, Statistical approach to Higgs boson couplings in the standard model effective field theory, Phys. Rev. D 97, 015007 (2018).

[34] R. Contino, M. Ghezzi, C. Grojean, M. Muhlleitner, and M. Spira, Effective Lagrangian for a light Higgs-like scalar, J. High Energy Phys. 07 (2013) 035.

[35] A. Alloul, B. Fuks, and V. Sanz, Phenomenology of the Higgs effective Lagrangian via FEYNRULES, J. High Energy Phys. 04 (2014) 110.

[36] P. Artoisenet et al., A framework for Higgs characterisation, J. High Energy Phys. 11 (2013) 043.

[37] O. Bruening and M. Klein, The Large Hadron Electron Collider, Mod. Phys. Lett. A 28, 1330011 (2013).

[38] J. L. A. Fernandez et al. (LHeC Study Group), A Large Hadron Electron Collider at CERN: Report on the physics and design concepts for machine and detector, J. Phys. G 39, 075001 (2012).

[39] J. L. A. Fernandez et al. (LHeC Study Group), On the relation of the LHeC and the LHC, arXiv:1211.5102.

[40] J. L. A. Fernandez et al., A large hadron electron collider at CERN, arXiv:1211.4831.

[41] M. Kumar, X. Ruan, R. Islam, A. S. Cornell, M. Klein, U. Klein, and B. Mellado, Probing anomalous couplings using di-Higgs production in electron-proton collisions, Phys. Lett. B 764, 247 (2017).

[42] A. Pomarol and F. Riva, Towards the ultimate SM fit to close in on Higgs physics, J. High Energy Phys. 01 (2014) 151.

[43] J. R. Ellis, M. K. Gaillard, and D. V. Nanopoulos, A phenomenological profile of the Higgs boson, Nucl. Phys. B106, 292 (1976).

[44] J. M. LoSecco, Higgs Boson production in neutrino scattering, Phys. Rev. D 14, 1352 (1976).

[45] Z. Hioki, S. Midorikawa, and H. Nishiura, Higgs Boson production in high-energy lepton-nucleon scattering, Prog. Theor. Phys. 69, 1484 (1983).

[46] J. Blumlein, G. J. van Oldenborgh, and R. Ruckl, QCD and QED corrections to Higgs boson production in charged current ep scattering, Nucl. Phys. B395, 35 (1993).

[47] T. Han and B. Mellado, Higgs Boson searches and the $H b \bar{b}$ coupling at the LHeC, Phys. Rev. D 82, 016009 (2010).

[48] S. S. Biswal, R. M. Godbole, B. Mellado, and S. Raychaudhuri, Azimuthal Angle Probe of Anomalous $H W W$ Couplings at a High Energy ep Collider, Phys. Rev. Lett. 109, 261801 (2012).
[49] H. Sun and X. Wang, Searches for the anomalous FCNC top-Higgs couplings at the LHeC, Eur. Phys. J. C 78, 281 (2018).

[50] X. Wang, H. Sun, and X. Luo, Searches for the anomalous FCNC top-Higgs couplings with polarized electron beam at the LHeC, Adv. High Energy Phys. 2017, 4693213 (2017).

[51] A. Senol, Anomalous Higgs couplings at the LHeC, Nucl. Phys. B873, 293 (2013).

[52] G. Passarino and M. Trott, The standard model effective field theory and next to leading order, arXiv:1610.08356.

[53] G. F. Giudice, C. Grojean, A. Pomarol, and R. Rattazzi, The strongly-interacting light Higgs, J. High Energy Phys. 06 (2007) 045.

[54] J. Ellis and T. You, Sensitivities of prospective future $e^{+} e^{-}$ colliders to decoupled new physics, J. High Energy Phys. 03 (2016) 089.

[55] N. D. Christensen and C. Duhr, FeynRules-Feynman rules made easy, Comput. Phys. Commun. 180, 1614 (2009).

[56] A. Alloul, N. D. Christensen, C. Degrande, C. Duhr, and B. Fuks, FeynRules 2.0-A complete toolbox for tree-level phenomenology, Comput. Phys. Commun. 185, 2250 (2014).

[57] C. Degrande, C. Duhr, B. Fuks, D. Grellscheid, O. Mattelaer, and T. Reiter, UFO-The universal FeynRules output, Comput. Phys. Commun. 183, 1201 (2012).

[58] J. Alwall, M. Herquet, F. Maltoni, O. Mattelaer, and T. Stelzer, MadGraph 5: Going beyond, J. High Energy Phys. 06 (2011) 128.

[59] J. Alwall, R. Frederix, S. Frixione, V. Hirschi, F. Maltoni, O. Mattelaer, H.-S. Shao, T. Stelzer, P. Torrielli, and M. Zaro, The automated computation of tree-level and next-toleading order differential cross sections, and their matching to parton shower simulations, J. High Energy Phys. 07 (2014) 079.

[60] J. Pumplin, D. R. Stump, J. Huston, H. L. Lai, P. M. Nadolsky, and W. K. Tung, New generation of parton distributions with uncertainties from global QCD analysis, J. High Energy Phys. 07 (2002) 012.

[61] B. Jager, Next-to-leading order QCD corrections to Higgs production at a future lepton-proton collider, Phys. Rev. D 81, 054018 (2010).

[62] P. Artoisenet, R. Frederix, O. Mattelaer, and R. Rietkerk, Automatic spin-entangled decays of heavy resonances in Monte Carlo simulations, J. High Energy Phys. 03 (2013) 015.

[63] S. Frixione, E. Laenen, P. Motylinski, and B. R. Webber, Angular correlations of lepton pairs from vector boson and top quark decays in Monte Carlo simulations, J. High Energy Phys. 04 (2007) 081.

[64] T. Sjostrand, L. Lonnblad, S. Mrenna, and P. Z. Skands, Pythia 6.3 physics and manual, arXiv:hep-ph/0308153.

[65] T. Sjostrand, S. Mrenna, and P.Z. Skands, A brief introduction to PYTHIA 8.1, Comput. Phys. Commun. 178, 852 (2008).

[66] M. Cacciari, G. P. Salam, and G. Soyez, FastJet user manual, Eur. Phys. J. C 72, 1896 (2012).

[67] G. Soyez, The SISCone, and anti- $k_{t}$ jet algorithms, arXiv:0807.0021. 
[68] R. Contino, A. Falkowski, F. Goertz, C. Grojean, and F. Riva, On the validity of the effective field theory approach to SM precision tests, J. High Energy Phys. 07 (2016) 144.

[69] C. Englert and M. Spannowsky, Effective theories and measurements at colliders, Phys. Lett. B 740, 8 (2015).

[70] M. Farina, G. Panico, D. Pappadopulo, J. T. Ruderman, R. Torre, and A. Wulzer, Energy helps accuracy: Electroweak precision tests at hadron colliders, Phys. Lett. B 772, 210 (2017).
[71] J. M. Butterworth, A. R. Davison, M. Rubin, and G. P. Salam, Jet Substructure as a New Higgs Search Channel at the LHC, Phys. Rev. Lett. 100, 242001 (2008).

[72] Y. L. Dokshitzer, G. D. Leder, S. Moretti, and B. R. Webber, Better jet clustering algorithms, J. High Energy Phys. 08 (1997) 001.

[73] M. Wobisch and T. Wengler, Hadronization corrections to jet cross-sections in deep inelastic scattering, arXiv:hep-ph/ 9907280 . 